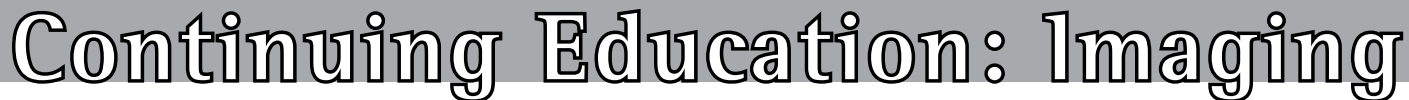

\section{Small interstitial nodules}

Pequenos nódulos intersticiais

\author{
Edson Marchiori ${ }^{1}$, Gláucia Zanetti², Bruno Hochhegger ${ }^{3}$
}

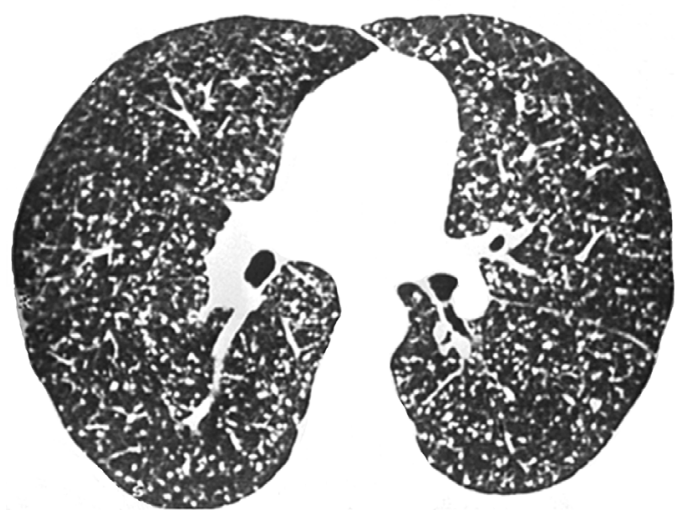

Figure 1 - Small nodules evenly distributed throughout the lungs, no particular lung compartment being predominantly affected. Note that some of the nodules touch the pleural surface, whereas others are in contact with the fissures.

A 45-year-old male patient presented to our outpatient clinic with a three-week history of low-grade fever, mildly productive cough, headache, and asthenia. He had lost $5 \mathrm{~kg}$ in the last four months. The patient reported that he had a brother who had been diagnosed with pulmonary tuberculosis and had been receiving treatment for it for two months. He also reported that he habitually spent his weekends on a grange in a rural area. A HRCT scan of the chest showed small disseminated nodules (Figure 1).

Multiple, small interstitial nodules constituted the only abnormal HRCT finding in our patient. Lung nodules appear as multiple round opacities that have soft tissue density and are smaller than $3 \mathrm{~cm}$. Small nodules (or micronodules) are those of less than $1 \mathrm{~cm}$ in diameter. On the basis of their distribution throughout the lung parenchyma, they can be classified as perilymphatic, centrilobular, or random.

Perilymphatic nodules are small nodules located predominantly in the peribronchovascular interstitium, interlobular septa, and subpleural regions (which contain the pulmonary lymphatic system). They are commonly found in cases of sarcoidosis, silicosis, and lymphangitic carcinomatosis. A centrilobular distribution is characterized by nodules that are located within a few millimeters of the pleural surface and fissures but do not touch them. Centrilobular nodules can be found in cases of hypersensitivity pneumonitis, silicosis, and infectious bronchiolitis, for example. A random distribution is characterized by small nodules randomly distributed in the secondary lobule and uniformly disseminated throughout the lungs. This distribution pattern is consistent with nodular disease that is disseminated through the blood, including metastasis and miliary granulomatous disease, particularly tuberculosis and histoplasmosis.

In the case reported here, the nodules were evenly distributed throughout the lungs, displaying a random pattern of distribution. This distribution pattern is consistent with miliary tuberculosis, histoplasmosis, and hematogenous metastasis. Metastatic nodules tend to predominate in the lower lung fields, whereas, in miliary tuberculosis, they tend to predominate in the upper lung fields. In addition, nodules commonly vary in size in cases of metastasis, whereas, in cases of miliary infection, they do not tend to. There are no CT criteria to aid in differentiating between miliary tuberculosis and histoplasmosis.

Our patient tested positive for HIV, his CD4 count was 140 cells/ $/ \mathrm{L}$, and radial immunodiffusion was positive for histoplasmosis. In the case reported here, a final diagnosis of histoplasmosis was established on the basis of the clinical picture, the epidemiological history, serology results, and CT findings.

\section{Recommended reading}

1. Webb WR, Muller NL, Naidich DP, editors. High-resolution CT of the lung. 4th ed. Philadelphia: Lippincott Williams \& Wilkins; 2008.

1. Full Professor Emeritus. Fluminense Federal University, Niterói; and Associate Professor of Radiology, Federal University of Rio de Janeiro, Rio de Janeiro, Brazil.

2. Professor. Graduate Program in Radiology, Federal University of Rio de Janeiro, Rio de Janeiro; and Professor of Clinical Medicine, Petrópolis School of Medicine, Petrópolis, Brazil.

3. Radiologist. Santa Casa Hospital Complex in Porto Alegre; and Professor of Radiology, Federal University of Health Sciences of Porto Alegre, Porto Alegre, Brazil. 